\title{
Apply traditional and molecular protocols for the detection of carrier state of visceral leishmaniasis in black Bengal goat
}

\author{
Sharmin Shahid Labony ${ }^{1}$, Nurjahan Begum ${ }^{1}$, Umme Kulsum Rima ${ }^{2}$, Md. Golam \\ Azam Chowdhury ${ }^{2}$, Mohammad Zakir Hossain ${ }^{2}$, Mohammad Ahsan Habib ${ }^{2}$, \\ Md. Abu Hadi Noor Ali Khan ${ }^{2}$ \\ 1(Department of Parasitology, Bangladesh Agricultural University, Bangladesh) \\ 2(Department of Pathology, Bangladesh Agricultural University, Bangladesh)
}

\begin{abstract}
Visceral leishmaniasis (VL) is the second largest parasitic killer of human in the world after malaria which is responsible for an estimated 500,000 new cases of VL in each year with 8-10\% mortality. The parasite is transmissible to humans and animals by the bite of phlebotomine sand fly. The clinical manifestations are highly diverse, humans and dogs are naturally infected, and the diseases are associated with several risk factors, yet to understand. The aims of this study were to apply traditional and molecular detection tools and more emphasis was given to identify goat as a carrier of visceral leishmaniasis. To demonstrate the promastigote and amastigote phases of Leishmania in tissues, traditional impression smear staining technique was used. For the confirmation of the species of Leishmania involved specific technique like polymerase chain reaction (PCR) was applied. A total of twenty goats were investigated and samples were tested using impression smear staining, histopathology and PCR. Blood smear and impression smears were prepared from spleen, liver, bone marrow and stained with Giemsa's stain. Using Giemsa's staining out of twenty goats investigated, six (6/20) were found to contain promastigote and amastigote stages of Leishmania in their visceral organs. Histopathological examination from the liver section of suspected goats showed degeneration, necrosis and non specific fibrous connective tissue proliferation compared to non-reactive goats. There was accumulation of macrophages in lymphoid follicles of spleen in five suspected goats. A highly sensitive and specific primers were used in PCR amplification with the extracted DNA from liver and spleen of suspected six goats. Results of PCR showed that two of them were generated 145bp amplicon selective for L. donovani in their liver and spleen. Leishmaniasis has a great public health significance and the protozoa found in goats of Fulbaria Upazilla may possess threat for transmission in human and other animals, require further investigation.
\end{abstract}

Key words: Black Bengal Goats, Visceral leishmaniasis, Giemsa's staining, Polymerase Chain Reaction (PCR)

\section{Introduction}

Leishmaniasis is a worldwide vector borne zoonotic disease caused by several species of the intracellular protozoan parasite. There are four forms of leishmaniasis including, visceral, cutaneous, diffuse cutaneous and mucocutaneous leishmaniasis [1]. Among them, visceral leishmaniasis remain in the top important vector borne zoonotic disease in many parts of the world and is caused by more than 20 species of the protozoan genus, Leishmania [2]. It is the second-largest parasitic killer in the world after malaria and responsible for an estimated 500,000 cases per year [3]. Visceral leishmaniasis (VL) is the most severe form of leishmaniasis and if left untreated, is usually fatal. Even with treatment, case fatality rates often exceed $10 \%$ in VL-endemic areas of Asia and Africa [4]. VL is a serious international public health problem affecting about 88 countries of the world, of which 66 being in the old world (Asia, Africa and Europe) and the remaining 22 in the new world (South \& Central America). Leishmaniasis is considered as one of the most neglected disease in Bangladesh based on the limited resources invested in diagnosis, treatment, control and its strong association with the poverty [5]. In Bangladesh, sporadic kala-azar cases were reported in 1970 and an outbreak occurred in Pabna district in 1980 [6]. This is one of the major health problem because 34 out of 64 districts are at risk, where $80 \%$ patients died within five years of affliction due to lack of diagnostic and treatment facilities in rural areas [7]. The true incidence of kala-azar in Bangladesh is not well documented but assumed to be close to 40, 000-45, 000 cases per year [8]. Ninety percent of total cases of VL in Bangladesh is reported in 10 districts during last 11 years (1994-2004) and of which more than 50\% of cases were reported in Mymensingh District. Out of 12 Upazilla of Mymensingh five were confirmedly reported to have kala-azar patients. Among these five, Fulbaria Upazilla showed the highest incidence and becoming epidemic in this area. Using the population of the respective Upazilla as the denominator, the incidence of kala-azar in Fulbaria Upazilla ranged from 30 to 33/10,000/year [8].

Also VL is an endemic disease of great public health importance in some rural communities of Bangladesh. Dog, cat, jackle [9], rodent, cattle, goat may act as a silent carrier and could play role in disease 
transmission in human. Detection of normal parasites in a clinical and subclinical sample is necessary to confirm a suspected case of leishmaniasis. Most commonly used methods to diagnose VL is to examine organs (liver and spleen) stained with modified Giemsa's [10]. PCR-based assays need to apply as highly sensitive and specific (upto 100\%) detection tool to diagnose Leishmania parasite irrespective of species or genus [11]. DNA from liver, spleen, lymphnode, blood and bone marrow smears stained with Giemsa,s stain are usually the most effortable primitive diagnosis tool [12]. It needs to adopt and correlate findings of traditional and molecular detection tool to understand the accuracy of detection techniques as well as the epidemiology of VL.

Goat may act as a carrier of VL but the possible role of goat for VL transmission has not been studied in Bangladesh. The asymptomatic goats in VL endemic area could carry organism in the liver and spleen but the authenticity of this findings has not confirmed yet. Therefore, this study was designed to standardize traditional and molecular tools for the detection of leishmanial protozoa in black bengal goat and identify goat as a carrier of VL in Fulbaria Upazilla, Mymensingh, Bangladesh.

\section{Materials And Methods}

A total of twenty goats of ill health and both sexes were randomly selected and collected from Fulbaria Upazilla, Mymensingh during the period from January to May, 2013. Among them, fifteen goat samples were collected from slaughter house of Fulbaria Upazilla and another five goat samples were collected from suspected cases. Systemic dissection and investigation were carried out and liver, spleen, blood and bone marrow were collected.

\subsection{Impression smears examination}

Thin smears of blood and impression smears from liver, spleen and bone marrow were made on to clean grease free glass slides, dried in air and fixed in ice cold absolute methanol (acetone free) for 15 minutes. The slides were dried in air and placed in Coplin jar containing working Giemsa's solution (Luna, 1968) and allowed to stain for 50 minutes. Slides were washed in running tape water for $30 \mathrm{sec}$, dried in air and examined at low (10x) and high power (40x and 100x) microscopic field. Extensive investigation was carried out for the presence of promastigote and amastigote stages of leishmanial protozoa in blood, in tissue spaces and in macrophages of various organs were employed and protozoal morphology was investigated and images were captured.

\subsection{Histopathological investigation}

During necropsy, portion of liver and spleen were collected and fixed in $10 \%$ buffered neutral formalin for histopathological studies. Formalin fixed tissue samples were trimmed, processed, sectioned and stained with H\&E (Luna, 1968). A low (10x) and high power (40x, 100x) microscopic investigation were carried out to observe the changes in the internal organs specific to VL.

\subsection{Detection of species of Leishmania by PCR \\ 2.3.1 DNA extraction}

For DNA extraction from the liver and spleen of goats Wizard ${ }^{1}$ Genomic DNA Purification Kit (Promega) was used according to the manufacturer's protocol. Briefly $100 \mu \mathrm{l}$ of $20 \%$ liver samples suspension was mixed with $600 \mu \mathrm{l}$ of nuclei lysis solution, incubated for $15 \mathrm{~min}$ at $65{ }^{\circ} \mathrm{C}$ temperature and $3 \mu \mathrm{l}$ of RNase Solution was added to the nuclear lysate and mixed by inversion. The mixture was incubated for 30 minutes at $37^{\circ} \mathrm{C}$. $200 \mu \mathrm{l}$ of Protein Precipitation solution was added and vortexed vigorously for $20 \mathrm{~s}$ and centrifuged at $13000 \mathrm{rpm}$ for $4 \mathrm{~min}$ and the precipitated protein was formed a tight white pellet. The supernatant was transferred to a new tube containing $600 \mu \mathrm{l}$ of room temperature isopropanol and centrifuged at $13000 \mathrm{rpm}$ for 1 min. The supernatant was discharged and $600 \mu \mathrm{l}$ of $70 \%$ ethanol was added. After the centrifugation at 13000 rpm for $1 \mathrm{~min}$, the ethanol was aspirated and the pellet air dried. The pellet was suspended in $100 \mu 1$ of the DNA rehydration solution. Then incubating at $65^{\circ} \mathrm{C}$ for 1 hour and finally stored the DNA at $4^{\circ} \mathrm{C}$. The DNA samples were evaluated quantitatively and qualitatively using spectrophotometry (A260 and A280) and agarose gel electrophoresis. One set of primers (Table 1) were used to identify the species of leishmanial protozoa.

\subsubsection{PCR}

PCR reactions were performed onto each DNA sample in $25 \mu \mathrm{l}$ reaction volume containing $7 \mu \mathrm{l}$ of Nuclease free water, $2 x$ PCR master mix (Promega Corporation, USA), $.25 \mu 1$ forward and $.25 \mu 1$ reverse primers and $5 \mu \mathrm{l}$ of DNA template. A total of 45 cycles of DNA amplification reaction for $L$. donovani was carried out. The thermal profile used for L. donovani comprised an initial denaturation for two minutes at $94^{\circ} \mathrm{C}$ followed by 45 cycles of DNA amplification reaction in a Master Cycler (Master Cycler Gradient, Eppendorf, Germany). The condition of PCR amplifications were denaturation for $60 \mathrm{sec}$ at $94^{\circ} \mathrm{C}$, primer annealing for $90 \mathrm{sec}$ at $62^{\circ} \mathrm{C}$ and extension for $30 \mathrm{sec}$ at $70^{\circ} \mathrm{C}$ followed by a final extension for $10 \mathrm{~min}$ at $70^{\circ} \mathrm{C}$. The PCR reactions were 
finally terminated by adding $3 \mu 150 \mathrm{mM}$ EDTA and PCR products were analyzed by electrophoresed in $2 \%$ agarose gel, stained with ethidium bromide and examined under UV light using an image documentation system (Cell Biosciences, Alphalmager HP, USA).

Table 1: Primers and their sequences for Leishmania donovani used in this study

\begin{tabular}{|l|l|c|c|}
\hline Primer & \multicolumn{1}{|c|}{ Sequence } & Product size & Reference \\
\hline LDF1(RV1) sense & 5'CTTTCTGGTCCCGCGGTAGG-3' & 145bp & [13] \\
\hline LDR1(RV2) antisense & 5'CCACCTGGCCTATTTTAACCA-3' & & \\
\hline
\end{tabular}

\section{Results And Discussion}

A total of twenty goats were examined in this study, where special fascination was focused to the detection of Leishmania protozoa in goats. For this, consequent studies on systemic necropsy of goats, blood smear and impression smears were performed from liver, spleen and bone marrow aspiration, systemic histopatholgy and PCR amplification was done using species (L. donovani) specific primers. In this study, Physical examination of the goats investigated revealed depression, loss of condition particularly decreased muscle mass over shoulders, hips, spine, serosanguineous nasal discharge, dull hair coat, spleenomegali and generalized lymphadenopathy. The goats were ill health, rough hair coat, alopecic but major gross changes characteristics of VL were not observed either in spleen, liver and bone marrow. However, in common the spleen appeared relatively larger. Blood smear and impression smears taken from the cut surface of liver, spleen and bone marrow stained with Giemsa's stain and were examined under light microscope. From this smear morphologically two forms of leishmanial stage was found, one is promastigote form and another is amastigote. Promastigotes are slightly elongated and contain a single nucleus with an anterior flagellum originating from a kinetoplast while amastigotes are slightly round to oval, still contain a single nucleus and kinetoplast, but retain only a rudimentary flagellum. In this study, pear or sperm head shaped leishmanial promastigote was seen in impression smear of liver (Figure 2a). Impression smear of spleen revealed highest number of promastigote (Figure 2c). Dark colored amastigote inside the ruptured macrophage was seen in bone marrow aspirates (Figure 1a). In blood (buffy coat) smear promastigote stage of Leishmania was also observed (Figure 1b). The concentration of leishmanial promastigotes (per 10x microscopic field) appeared higher in the impression smears prepared from spleen $(\mathrm{N}=5-15)$, followed by liver $(\mathrm{N}=5-7)$, bone marrow $(\mathrm{N}=3)$. Using smear microscopy, the species of Leishmania involved whether pathogenic or non pathogenic remain undetected. However, comparative data on to the morphologic analysis of the developmental stages of leishmanial protozoa in man and animals is very limited. [14] investigated clinically suspected cases of visceral leishmaniasis $(83.4 \%)$ in canids and identified positive cases by examining bone marrow. However studies showed that bone marrow examination was the least sensitive method for the detection of visceral leishmaniasis [15], [16], [17]. This study showed that impression smears prepared from sternum and stained with Giemsa's showed promastigotes, however, the number per 10x microscopic field were relatively lower compared to the load of promastigotes in spleen and liver. The load of promastigotes per microscopic field in the impression smears of spleen were always appeared higher compared to other organs.

Histopathological examination was done in liver and spleen of six suspected goats. The results of histopathological examination revealed numerous rod shaped structure similar to promastigote stages of Leishmania in the liver. Numerous dark color bodies in Vonkupffer cell of liver was seen (Figure 3a) and these could be the amastigote stages of the protozoa. Histopathological examination of spleen revealed nodule like structure (Figure 3b) and containing infiltration of macrophage in lymphoid follicle. Notable changes in the bone marrow was not seen in this study. Histopathological study did not reveal much amastigote stage of protozoa in the macrophages of liver, spleen, blood and bone marrow. The presence of large number of promastigote in liver and spleen without tissue reaction and lacking of inflammation indicate the role of goats as a asymptomatic carrier of VL. Scatterdly distributed granulomatus reaction were seen in liver parenchyma. The nodule of hepatic parenchyma contain infiltration of macrophage. There was lacking of caseous necrotic centre. Fibrotic patches were well documented along the hepatic parenchyma could provoke by dispatching of leishmanial promastigotes. There were numerous darkly stained promastigotes dispatched congested central vein indicating stage of disseminating of the protozoa. [18] reported the reason of the granuloma formation and presence of necrosis or proliferation of connective tissues and giant cells in chronically infected liver. There was a infiltration of macrophage in lymphoid follicle of spleen and nodule like structure was also seen. Early lesions with high parasitic load can show granuloma at the edge of lesions and deeper sections of the biopsy [19]. [20] described granulomatous leishmanial lesions in monkey, consisted of macrophages containing parasites, lymphocytes, plasma cells, and occasionally eosinophils. Differences were not observed between promastigote or amastigote derived nodular lesion or strains of leishmanial protozoa involved, course of infection and variation in either the sex and ages of the hosts and geography.

Many techniques such as isoenzyme electrophoresis, monoclonal antibodies, in situ hybridization, molecular karyotyping and restriction enzyme are currently used to detect Leishmania compared to PCR. These 
techniques require a larger quantity of sample material, involve a lengthy technical process and may present difficulties in the interpretation of results [21], [22], [23]. PCR assays have greatly improved the sensitivity and specificity to diagnose leishmanial infection in canids [24]. However, to make this technique achievable, the sampling methods need to be noninvasive, easy and painless. Using PCR on to the DNA from Conjunctival swab diagnosed symptomatic dogs successfully and observed $92 \%$ sensitivity [25]. However, [26] found $91.7 \%$ and [27] obtained between 73.9 and $95.6 \%$ sensitivity depending upon the PCR method used to diagnose VL. In this study as the animals were examined at necropsy and from slaughter house, PCR amplification (Table 1) with the DNA template from the liver and spleen of goats were carried out (Figure 4) using primers specific for L. donovani (Figure 4). PCR amplification with the DNA from these organs showed $145 \mathrm{bp}$ amplicon indicating infectivity of goats with $L$. donovani. This study provide evidence that the goats $(\mathrm{N}=20)$ at Fulbaria Upazilla were infected with $L$. donovani. Molecular detection using PCR to investigate the carrier state of VL in goats was reported by [28] where spleenomegali and fibrotic condition of liver were well demarcation of leishmaniasis. As there was lacking data regarding infectivity of goats with Leishmania, to the best of our knowledge may be this is the first study in Bangladesh describing occurrence of VL in domestic small ruminants. The result may come to an assumption that goats can be considered as an important carrier for leishmanial infection similar to other wild canids [29].
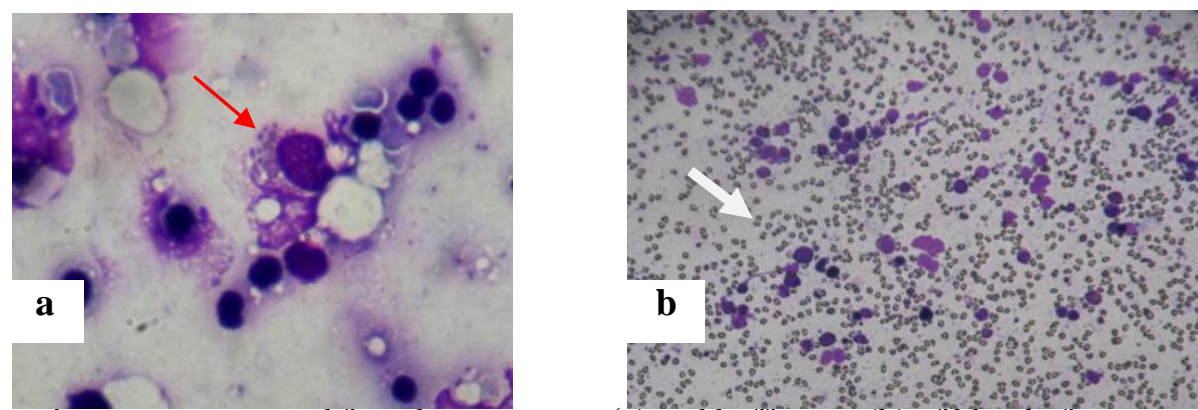

Fig.1 Impression smears prepared trom bone marrow (a) and butfy coat (b) of blood of a goat and stained with

Giemsa's. Dark colored numerous amastigotes (red arrow, $100 \mathrm{X}$, left) was seen inside the ruptured

macrophage. Promastigote (white arrow, $10 \mathrm{X}$, right) phase of Leishmania protozoa was seen in the smear prepared from blood sample.

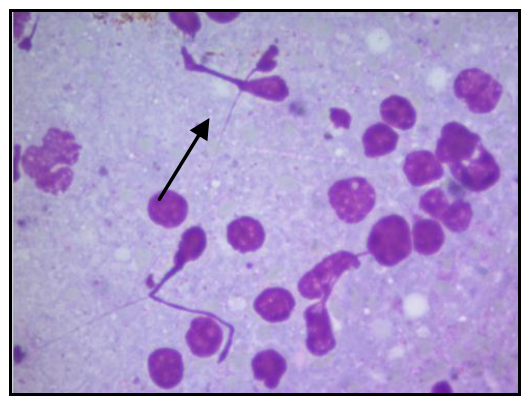

a $(100 X)$

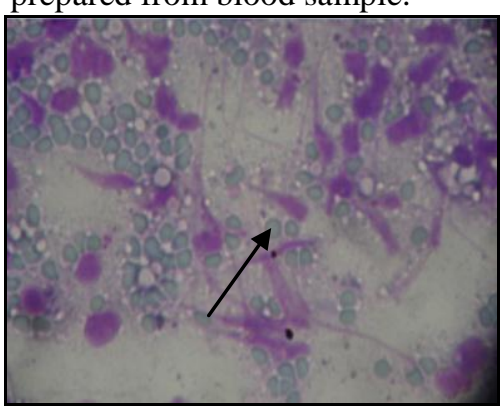

$\mathrm{b}(100 \mathrm{X})$

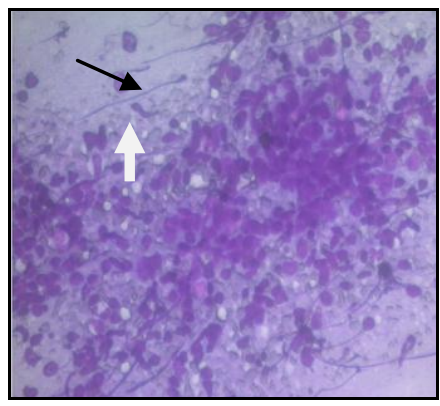

$\mathrm{c}(40 \mathrm{X})$

Fig.2 Morphological investigation of promastigote stages of leishmanial protozoa (black arrow) in different organs impression smears prepared from goat and stained with Giemsa's. The promastigote stage of the parasite was seen in the intercellular spaces and found to contain a tail (white arrow) and a head (black arrow) with variable morphology. Under oil immersion microscopy ballon shaped (a, black arrow), sperm head shaped (b,

black arrow) and sometimes tadpole shaped (c, black arrow) appearance of the protozoa were seen.
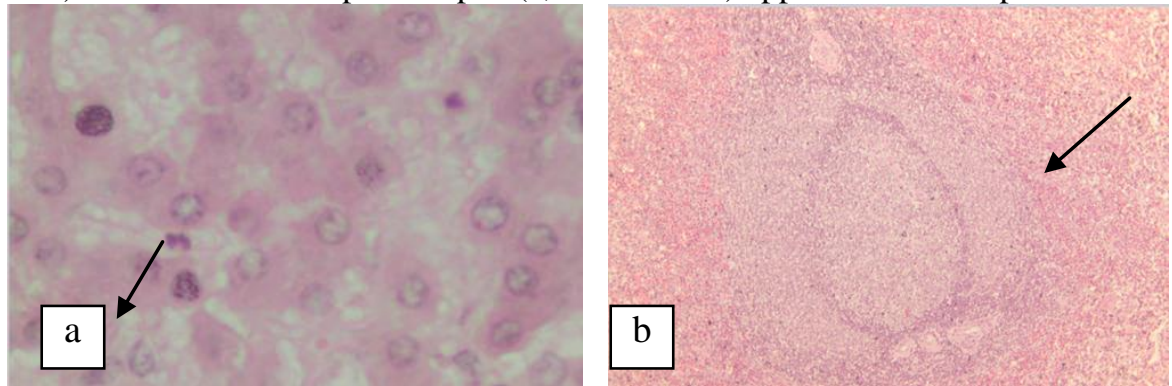

Fig.3 Section of the liver and spleen of a goats ( $\mathrm{a} \& \mathrm{~b})$ and stained with H\&E. Dark color amsatigote was seen (black arrow, 100X, left) in Vonkupffer cell of liver section (a). Scatterdly distributed granulomatus reaction were seen in liver parenchyma. The nodule of hepatic parenchyma contain infiltration of macrophage and closely packed collection of macrophagess replacing hepatic parenchyma and lacking caseous necrotic centre. Nodule like structure (black arrow, 10X, right) in lymphoid follicle of spleen was seen. 


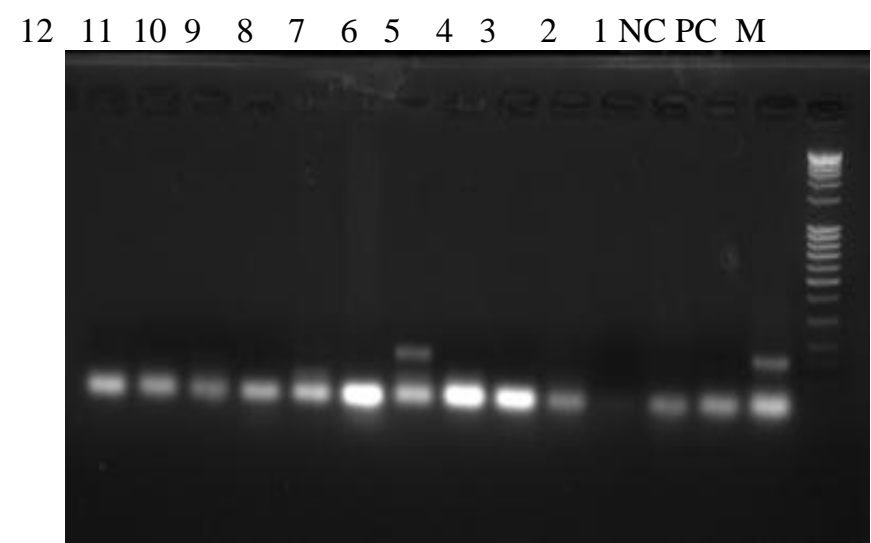

Fig.4 Agarose gel electrophoresis of PCR products with the genomic DNA obtained from the visceral organs of goats. Leishmania donovani specific primers were found to amplify genomic DNA of Leishmania in PCR settings. Lane - 1, 2, 3, 4, 5, 6 containing DNA template from the liver of goats and Lane - 7, 8, 9, 10, 11, 12 containing DNA template from the spleen of goats. Lane - M containing 100bp DNA ladder, Lane - NC is for Negative control, Lane - PC is for Positive control. PCR amplification showed the presence of $L$. donovani in goats 6 and 8 by generating 145 bp amplicon.

\section{Conclusions}

This study concludes that probably this is the first study in Bangladesh detecting Leishmania sp. in goats by using PCR. However further studies are needed by increases sample size \& to identify genomic DNA in sand flies by using PCR which transmitting VL to other animals and human beings.

\section{Acknowledgement}

The research was conducted under the project of Bangladesh Agricultural Research Council (SPGR, PIU-BARC, NATP Phase-1,307).

\section{References}

[1] C.A.P. Tavares, A.P. Fernandes and M.N. Melo, Molecular diagnosis of leishmaniasis. Expert Molecular Diagnosis, 3, 2003, 657667.

[2] O. Courtenay, E.W. Santana, P.J. Johnson, I.A. Vasconcelos, A.W. Vasconcelos, Visceral leishmaniasis in the hoary zorro, 1996

[3] P. Desjeux, "The increase of risk factors for leishmaniasis worldwide". Transactions of the Royal Society of Tropical Medicine and Hygiene, 95, 2001, 239-243.

[4] J.D. Berman, Human leishmaniasis: clinical, diagnostic, and chemotherapeutic developments in the last 10 years. Clinical Infectious Diseases, 24,1997, 684-703.

[5] J. Alvar, S. Yactayo and C. Bern, Leishmaniasis and poverty. Trends in Parasitology, 22, 2006, 552-557.

[6] M. Elias, A.J. Rahman, N.I. Khan, Visceral leishmaniasis and its control in Bangladesh. Bull World Health Organization, 67, 1989, 43-49.

[7] N.I. Hasib, The Independent of Bangladesh. 2004,12-28-2004 URL: http://independent- bangladesh.com/).

[8] C. Bern and R. Chowdhury, The epidemiology of visceral leishmaniasis in Bangladesh prospects for improved control. Indian Journal of Medical Research, 123, 2006, 275-288.

[9] M.A.H.N.A. Khan, M.S.S Khan, J. Bashu, U.K. Rima, M. Pervin, M.Z. Hossain, M.A. Habib , G.A. Chowdhury and M.M. Hossain, Visceral leishmaniasis is endemic in golden jackals of bangladesh agricultural university campus, a threat for expanding future zoonotic visceral leishmaniasis. Bangladesh Journal of Veterinary Medicine 10 (1\&2), 2012, 101-109.

[10] B.L. Herwaldt, Leishmaniasis. Lancet 354, 1999, 1191-1199.

[11] O.F. Osman, L. Oskam, E.E. Zijlstra, A.M. Hassan, D.A. Naeim, P.A. Kager, Use of the polymerase chain reaction to assess the success of visceral leishmaniasis treatment. Transactions Royal Society Tropical Medicine Hygiene, 92, 1998, 397-400.

[12] R.W. Ashford, M.A. Bray, M.P. Hutchnson and R.S. Bray, The epidemiology of cutaneous leishmaniasis in Ethiopia. Transactions Research society of Tropical Medicine Hygiene, 67,1973, 568-601.

[13] A.H.S. Gomes, I.M.R. Ferreira, M.L.S.R. Lima, E.A. Cunha, A.S. Garcia, M.F.L. Araujo and V.L. Pereira-Chioccola, PCR identification of Leishmania in diagnosis and control of canine leishmaniasis. Veterinary Parasitology, 144, $2006,234-241$.

[14] F. Al Shanawi, Comparison of the course of laboratory infection in Iraqi strain of Lei Alvar shmania donovani. MSc Thesis, College of Science, University of Baghdad, 1975.

[15] E.E. Zijlstre, M.S. Ali, A.M. El Hassan, I.A. El Toum and M. Satti, Kala azar: a comparative study of parasitological methods and the direct agglutination test in diagnosis. Transaction of the Royal Society of Tropical Medicine and Hygiene, 86, $1992,505$.

[16] A.H. Jassim, The evaluation and application of locally prepared direct agglutination test for the diagnosis and sero epidemiology of visceral leishmaniasis in Basrah. PhD Thesis, College of Medicine, University of Mustansiryia, Iraq, 1998.

[17] A. Ataya and N.A. Sarji, Comparative study of methods used for the diagnosis of leishmaniasis in Syria. Journal of the Arab Board of Medicine ,3, 2001,103.

[18] L.Tryphonas, Z. Zawidzka, M.A. Bernard, E.A. Janzen, Visceral Leishmaniasis in dog: clinical, Hematological and Pathological observations. Canadian Journal of comparative Medicine, 41, 1977, 1-12.

[19] N.S. Mansur, Stauber L.A. , J.R. McCoy, Leishmaniasis in the Sudan republic. Comparison and epidemiology implications of experimental canine infections with Sudanese, Mediterranean and Kenyan strains of Leishmania donovani Journal of Parasitology, $56,1970,468-472$ 
[20] R. Lujan, W.L. Chapman, W.L. Hanson, V.A. Dennis, Leishmania braziliensis: Development of primary and satellite lesions in the experimentally infected owl monkey, Aotus trivirgatus. Experimental Parasitology 61(3), 1986, 348-358.

[21] T. Mimori, J. Sasaki, M. Nakata, E.A. Gomez, H. Uezato, S. Nonaka, Rapid identification of Leishmania species from formalinfixed biopsy samples by polymorphism-specific polymerase chain reaction. Gene, 210, 1998, 179-186.

[22] R. Reithinger, J.C. Dujardin, H.C. Louzir, B. Pirmez, S. Brooker, Cutaneous leishmaniasis. Lancet Infectious Disease, 7, 2007, 581-596.

[23] F.T. Silveira, R. Lainson, C.E. Corbett, Clinical and immunopathological spectrum of American cutaneous leishmaniasis with special reference to the disease in Amazonian Brazil: a review. Mem Inst Oswaldo Cruz, 99, 2004, 239-251.

[24] D.F.Torres, The role of dogs as reservoirs of Leishmania parasites, with emphasis on Leishmania infantum and Leishmania (Viannia) braziliensis. Veterinary Parasitology, 149, 2007, 139-146.

[25] A.D. Strauss, L.J. Charles, B. Ofer, G. Liat and B. Gad, Polymerase chain reaction using noninvasively obtained samples, for the detection of Leishmania infantum DNA in dogs. The Journal of Infectious Diseases, 189(9), 2004, 1729-1733.

[26] S.A. Ferreira, L.T. Ituassu, M.N. de Melo and A.S.R. de Andrade, Evaluation of the conjunctival swab for canine visceral leishmaniasis diagnosis by PCR hybridization in Minas Gerais State, Brazil. Veterinary Parasitology, 152(34), $2008,257-263$.

[27] M.M. Pilatti, S.A. Ferreira, M.N. de Melo and A.S.R. de Andrade, Comparison of PCR methods for diagnosis of canine visceral leishmaniasis in conjunctival swab samples. Research in Veterinary Science, 87(2), 2009, 255-257.

[28] A.F. Koutinas, V. Kontos, H. Kalidrimidou and S. Lekkas, Canine leishmaniasis- associated nephropathy: A clinical, clinicopathological and pathologic study in 14 spontaneous cases with proteinuria. European Journal Animal Practice, 5, 1995, 3138.

[29] E.M. Michalsky, M.F. Rocha, A.C.V.M. da Rocha Lima, J.C. França Silva, PiresM.Q., F.S. Oliveira, R.S. Pacheco, S.L. dos Santos, R.A. Barata, A.J. Romanha and C.L.Fortes Dias, Infectivity of seropositive dogs, showing different clinical forms of leishmaniasis, to Lutzomyia longipalpis phlebotomine sand flies. Veterinary Parasitology, 147(12), 2007, 67-76. 